\title{
ÍNDICE DE DESENVOLVIMENTO HUMANO E QUALIDADE DE VIDA DE IDOSOS FREQUENTADORES DE UNIVERSIDADES ABERTAS PARA A TERCEIRA IDADE
}

\author{
INDICE DE DESARROLLO HUMANO Y CALIDAD DE VIDA DE LAS \\ PERSONAS MAYORES ASISTENTES DE UNIVERSIDADES ABIERTAS PARA LA \\ TERCERA EDAD \\ HUMAN DEVELOPMENT INDEX AND QUALITY OF LIFE OF ELDERLY \\ ATTENDING UNIVERSITIES OF THE THIRD AGE
}

\author{
Nathaly Wehbe Dawalibi, Rita Maria Monteiro Goulart, Rita de Cássia de Aquino, Carla Witter, \\ Marcelo de Almeida Buriti \\ Universidade São Judas Tadeu, São Paulo/SP, Brasil \\ e Leandro Campi Prearo \\ Universidade Municipal de São Caetano do Sul, São Caetano do Sul/SP, Brasil
}

\begin{abstract}
RESUMO
Verificou-se a relação entre "desenvolvimento humano" e qualidade de vida de idosos em um estudo transversal, quantitativo-descritivo de campo, com 182 idosos, de ambos os gêneros, $\geq 60$ anos, frequentadores de Universidades Abertas para a Terceira Idade, dos municípios A, B e C. A qualidade de vida foi avaliada pelo WHOQOL - BREF. Foram utilizados os testes estatísticos Kruskal-Wallis e Mann-Whitney, adotando-se nível de significância de 5\%. Não houve diferença estatisticamente significante quando comparada a qualidade de vida dos idosos dos três municípios entre si; houve no domínio ambiental comparando-se A com os outros municípios; e nos domínios social e ambiental comparando-se A com C. Concluiu-se que o "desenvolvimento humano", medido pelo IDH/IDHM, exerceu influência sobre a qualidade de vida dos idosos nos domínios social e ambiental, sendo que os melhores resultados foram obtidos entre os habitantes dos municípios A e B, em comparação aos de C.
\end{abstract}

Palavras-chave: idosos; qualidade de vida; índice de desenvolvimento humano.

\section{RESUMEN}

Investigamos la relación entre el "desarrollo humano" y la calidad de vida de las personas mayores en estudio de campo transversal, cuantitativo-descriptivo, con 182 ancianos de ambos sexos, $\geq 60$ años, asistentes de Universidades Abiertas para la Tercera Edad, de las ciudades A, B y C. La calidad de vida se evaluó por el WHOQOL - BREF. Se utilizaron pruebas estadísticas Kruskal-Wallis y Mann-Whitney, nivel de significación de $5 \%$. No se encontraron diferencias significativas cuando se comparan la calidad de vida en los tres municipios juntos; existió en el domínio ambiental mediante la comparación de A con los otros municipios y nos domínios social y ambiental, mediante comparación de A con C. El "desarrollo humano", medido por el IDH/IDHM, ejerció una influencia en la calidad de vida en las cuestiones sociales y ambientales. Los mejores resultados se obtuvieron entre los habitantes de A y B en comparación con C.

Palabras clave: personas mayores; calidad de vida; índice de desarrollo humano.

\begin{abstract}
We investigated the relationship between "human development" and quality of life of elderly in a cross-sectional, quantitative-descriptive study, with 182 elderly of both genders, $\geq 60$ years, attending the Universities of the Third Age, in districts A, B, C. The quality of life was evaluated by WHOQOL-BREF. Kruskal-Wallis and Mann-Whitney statistical tests were applied, considering a significance level of $5 \%$. There was no statistically significant difference when comparing the quality of life of the three districts together; there was in environmental domain when comparing A with the other districts; and in social and environmental domains when comparing A with C. It was concluded that the "human development", measured by HDI, influenced on the quality of life of elderly in social and environmental domains, and the best results were obtained among the inhabitants of $\mathrm{A}$ and $\mathrm{B}$, compared to the inhabitants of $\mathrm{C}$.
\end{abstract}

Keywords: elderly; quality of life; human development index. 


\section{Introdução}

O Índice de Desenvolvimento Humano (IDH) é um indicador comparativo usado para segmentar os países desenvolvidos (elevado desenvolvimento humano), em desenvolvimento (desenvolvimento humano médio) e subdesenvolvidos (desenvolvimento humano baixo). O cálculo do IDH é composto a partir de dados de expectativa de vida ao nascer, educação e produto interno bruto (PIB) per capita (Programa das Nações Unidas para o Desenvolvimento - PNUD, 2012).

Esse índice foi criado no início da década de 1990, pela Organização das Nações Unidas (ONU), para se contrapor ao PIB per capita, que considera apenas a dimensão econômica do desenvolvimento (PNUD, 2003). Atualmente, ainda é a medida de desenvolvimento humano mais conhecida e utilizada em todo o mundo (Torres, Ferreira, \& Dini, 2003).

A expectativa de vida ao nascer reflete as condições de saúde da população, a educação leva em conta a taxa de alfabetização de adultos e a taxa combinada de matrícula nos níveis fundamental, médio e superior, e a renda é medida pelo poder de compra da população, baseada no PIB per capita ajustado ao custo de vida local, por meio da metodologia conhecida como Paridade do Poder de Compra (PCC) (Scarpin \& Slomski, 2007).

O resultado é ordenado segundo valores obtidos no cálculo, podendo ir de 0 (pior situação de desenvolvimento humano) até 1 (melhor situação de desenvolvimento humano). Conforme a ONU, a região ou o país apresenta alto desenvolvimento humano se o IDH for $\geq 0,8$; médio, de 0,79 a 0,5 ; e baixo, se for $\leq$ 0,49 (PNUD, 2003; Sen, 2000; Silva $\&$ Panhoca, 2007).

O IDH brasileiro passou de 0,788 , em 2003, para $0,792 \mathrm{em} 2004$, resultado que mantinha o país entre as 83 nações de médio desenvolvimento humano, e ausente, portanto, do grupo de 63 nações de alto desenvolvimento humano, que tem a Noruega no topo pelo sexto ano consecutivo (IDH de 0,965 ) (PNUD, 2006).

O relatório do IDH para 2010 mostra o Brasil na $73^{a}$ posição entre 169 países, com IDH de 0,699, situando-se entre os países de alto desenvolvimento humano, a partir da adoção da nova metodologia. Os cinco primeiros colocados são, pela ordem, Noruega, Austrália, Nova Zelândia, Estados Unidos e Irlanda. O cinco últimos são Zimbábue, República Democrática do Congo, Níger, Mali e Burkina Faso, respectivamente (PNUD, 2010).
Classificado pela ONU como um país intermediário quanto à renda per capita, o Brasil possui o segundo maior índice de concentração de renda do mundo, sendo superado apenas por Suazilândia, na África. No Brasil, 50\% dos mais pobres ganham o equivalente ao que ganham $1 \%$ dos mais ricos (Pinheiro, Carvalho, \& Freitas, 2009).

De acordo com Barros, Henriques e Mendonça (2001), o Brasil exibe níveis de pobreza superiores à maioria dos países que têm renda per capita semelhante à sua. Isso porque, apesar de ser um país de produtividade crescente, apresenta distribuição de renda muito desigual.

Ao se avaliar como vivem as pessoas de uma região ou de um país, mais do que conhecer sua renda, deve-se ter a preocupação de saber como esse dinheiro é distribuído e se ele permite o atendimento às suas necessidades básicas e o acesso a serviços médicos de qualidade e a uma boa educação. Além disso, deve-se levar em conta se as pessoas desfrutam de condições dignas de trabalho, se são amparadas pela lei e se possuem uma vida social e um suporte familiar satisfatórios, fatores essenciais quando se pensa em dignidade humana (Torres et al., 2003).

O conceito de qualidade de vida é amplo e subjetivo e abrange uma grande gama de aspectos, tais como: o bem-estar pessoal, a autoestima, a capacidade funcional, o nível socioeconômico, o estado emocional, a interação social, a atividade intelectual, o autocuidado, o suporte familiar, o estado de saúde, os valores culturais, éticos e a religiosidade, o estilo de vida, a satisfação com o emprego e/ou com as atividades da vida diária e com o ambiente em que se vive (Neri, 2007a; Vecchia, Ruiz, Bocchi, \& Corrente, 2005).

Segundo o Grupo de Qualidade de Vida da Organização Mundial da Saúde (OMS), qualidade de vida refere-se à "percepção do indivíduo de sua posição na vida, no contexto da cultura e sistema de valores nos quais ele vive, em relação aos seus objetivos, expectativas, padrões e preocupações" (WHOQOL Group, 1994, citado por Grupo de Estudos em Qualidade de Vida, 2008, s.p.). Com base nesse conceito, a OMS desenvolveu um instrumento, intitulado WHOQOL $-100^{1}$, que valoriza a percepção subjetiva do indivíduo, ao contrário de outros instrumentos que adotam a percepção objetiva do sujeito Tal instrumento caracteriza-se por ser multidimensional e possui aspectos positivos e negativos. Ainda, houve a preocupação de se utilizar uma metodologia genuinamente transcultural, possibilitando sua utilização em diferentes culturas com mais adequação (Fleck et al., 2008). 
O WHOQOL - 100 foi desenvolvido com a participação de 15 centros em localizações geográficas distintas, com situações socioeconômicas, de desenvolvimento social e contextos culturais diferentes. Os mencionados centros participaram de todas as etapas de desenvolvimento do instrumento, incluindo a composição dos domínios, a escolha das formulações a serem utilizadas e os testes-piloto e de campo (Fleck et al., 2008).

Posteriormente, a OMS elaborou uma versão abreviada do WHOQOL - 100, o WHOQOL - BREF, que contém quatro domínios (físico, psicológico, social e ambiental), além de duas questões de autopercepção sobre qualidade de vida. Isso porque o WHOQOL - 100 é muito longo e inviável para ser usado em estudos de bases populacionais e epidemiológicas, ou em conjunto com outras medidas clínicas (Fleck et al., 2008).

Trabalhando com a hipótese de que quanto maior o IDH de um país ou uma região, melhor a qualidade de vida de seus habitantes, o objetivo do presente estudo foi verificar a relação entre o "desenvolvimento humano", medido pelo IDH e IDHM (Índice de Desenvolvimento Humano Municipal), e a qualidade de vida, medida pelo WHOQOL - BREF, de idosos frequentadores de Universidades Abertas para a Terceira Idade em três municípios do Estado de São Paulo.

\section{Método}

Trata-se de um estudo transversal, do tipo quantitativo-descritivo de campo, do qual participaram 182 idosos, de ambos os gêneros, com idades maior ou igual a 60 anos, frequentadores de Universidades Abertas para a Terceira Idade, de três municípios do Estado de São Paulo: São Caetano do Sul, São Paulo e Mogi das Cruzes, nesta pesquisa designados de A, B e C, respectivamente. Segundo o IBGE (2010), o IDHM de A é 0,862 ; de B é 0,805 ; e de C é 0,783 .

Em 2012, o PNUD Brasil, o Instituto de Pesquisa Econômica Aplicada (IPEA) e a Fundação João Pinheiro (FJP) assumiram o desafio de adaptar a metodologia do IDH global para calcular o IDHM dos 5.565 municípios brasileiros a partir de dados do Censo Demográfico de 2010. ... O IDHM brasileiro segue as mesmas três dimensões do IDH Global - longevidade, educação e renda, mas vai além: adequa a metodologia global ao contexto brasileiro e à disponibilidade de indicadores nacionais. Embora meçam os mesmos fenômenos, os indicadores levados em conta no IDHM são mais adequados para avaliar o desenvolvimento dos municípios brasileiros. (PNUD, IPEA, \& FJP, 2012, s.p.)
Por tal motivo, foi escolhido o IDHM para este estudo.

$\mathrm{Na}$ primeira consulta com as nutricionistas/ pesquisadoras das três universidades, que ocorreu quando os idosos estavam ingressando nos programas para a terceira idade, foi aplicada uma anamnese inicial, a fim de se obter os dados sociodemográficos dos participantes.

As variáveis estudadas foram: gênero, município de residência, idade, estado civil, ter ou não filhos, morar sozinho ou acompanhado, nível de escolaridade, renda familiar mensal e trabalho/aposentadoria.

A qualidade de vida dos idosos foi avaliada por meio da aplicação do World Health Organization Quality of Life Questionnaire (WHOQOL - BREF), nos domínios físico, psicológico, social e ambiental. Além desses domínios, o questionário contém duas questões de autopercepção sobre qualidade de vida (Fleck et al., 2008).

A aplicação do WHOQOL - BREF também ocorreu na primeira consulta, sendo que as nutricionistas/pesquisadoras das respectivas universidades apenas assistiram os idosos em caso de necessidade de esclarecimento em enunciados de algumas questões, sem, no entanto, interferir em suas respostas. Para os que não mostraram quaisquer dúvidas, o questionário foi respondido sem ajuda.

A questão 1 de autopercepção diz respeito ao que o idoso acha da sua vida, tendo em mente seus valores, prazeres, suas aspirações e preocupações. A questão 2 refere-se a quanto o idoso recebe dos outros o apoio de que necessita, segundo a percepção do próprio idoso.

Cada domínio apresenta uma escala de zero a 100. Não existem pontos de corte para melhor ou pior qualidade de vida no WHOQOL - BREF. Assim, melhor qualidade de vida é indicada por valores mais próximos a 100 , e pior qualidade de vida, por valores mais próximos a zero.

Para verificar se havia diferença estatisticamente significante na qualidade de vida dos idosos nos três municípios, foi aplicado o teste de Kruskal-Wallis, assim como sugerem Siegel e Castellan Junior (2006), para amostras divididas em três ou mais grupos (três municípios, no caso deste estudo) e para amostras não aderentes à distribuição normal.

Ainda, para verificar diferenças na qualidade de vida dos idosos nos municípios dois a dois, foi utilizado o teste de Mann-Whitney, o qual, segundo Siegel e Castellan Junior (2006), é mais adequado para a comparação de dois grupos. 
Registre-se que para todas as análises considerouse o nível de significância de $5 \%(\mathrm{p} \leq 0,05)$.

\section{Resultados}

A amostra estudada foi constituída, salários mínimos mensais $(39,6 \%)$, não trabalhavam predominantemente, por idosos do gênero feminino $\quad(89,0 \%)$ e estavam aposentados $(80,2 \%)$ (Tabela 1$)$.

$(89,6 \%)$, com idade média de $67 \pm 6,3$ anos, casados $(47,8 \%)$, com pelo menos um filho $(86,8 \%)$ e que moravam acompanhados (75,8\%). Observou-se maior frequência de idosos que completaram o ensino médio $(43,4 \%)$, possuíam renda familiar entre um e três salários mínimos mensais $(39,6 \%)$, não trabalhavam
$(89,0 \%)$ e estavam aposentados $(80,2 \%)$ (Tabela 1$)$.

Tabela 1. Distribuição percentual de idosos matriculados em programas para a terceira idade de universidades localizadas no Estado de São Paulo, segundo as características sociodemográficas

\begin{tabular}{|c|c|c|}
\hline Características sociodemográficas & $\mathbf{N}$ & $\%$ \\
\hline \multicolumn{3}{|l|}{ Gênero } \\
\hline Masculino & 019 & 10,4 \\
\hline Feminino & 163 & 89,6 \\
\hline \multicolumn{3}{|l|}{ Município de residência } \\
\hline São Caetano do Sul & 106 & 58,2 \\
\hline Mogi das Cruzes & 045 & 24,8 \\
\hline São Paulo & 031 & 17,0 \\
\hline \multicolumn{3}{|l|}{ Idade (anos) } \\
\hline $60-69$ & 125 & 68,7 \\
\hline $70-79$ & 047 & 25,8 \\
\hline $80-89$ & 010 & 5,5 \\
\hline \multicolumn{3}{|l|}{ Estado civil } \\
\hline Casado & 87 & 47,8 \\
\hline Viúvo & 64 & 35,2 \\
\hline Solteiro & 16 & 8,8 \\
\hline Divorciado & 15 & 8,2 \\
\hline \multicolumn{3}{|l|}{ Filhos } \\
\hline Sim & 158 & 86,8 \\
\hline Não & 024 & 13,2 \\
\hline \multicolumn{3}{|l|}{ Mora sozinho } \\
\hline Sim & 044 & 24,2 \\
\hline Não & 138 & 75,8 \\
\hline \multicolumn{3}{|l|}{ Escolaridade } \\
\hline Ensino Fundamental & 070 & 38,5 \\
\hline Ensino Médio & 079 & 43,4 \\
\hline Ensino Superior & 033 & 18,1 \\
\hline \multicolumn{3}{|l|}{ Renda familiar mensal (s.m.*) } \\
\hline$<1$ & 022 & 12,1 \\
\hline$\geq 1 \mathrm{e}<3$ & 072 & 39,6 \\
\hline$\geq 3$ e $<5$ & 061 & 33,5 \\
\hline$\geq 5 \mathrm{e}<10$ & 019 & 10,4 \\
\hline$\geq 10$ & 007 & 3,8 \\
\hline Sem informação & 001 & 0,6 \\
\hline \multicolumn{3}{|l|}{ Trabalha } \\
\hline Sim & 020 & 11,0 \\
\hline Não & 162 & 89,0 \\
\hline \multicolumn{3}{|l|}{ Aposentado } \\
\hline Sim & 146 & 80,2 \\
\hline Não & 036 & 19,8 \\
\hline
\end{tabular}

*s.m.: salário mínimo

\section{.}


Na Tabela 2, são apresentados os resultados em relação aos domínios e às questões de autopercepção sobre qualidade de vida dos idosos, nos três municípios estudados. Como pode ser verificado, não foram encontradas diferenças estatisticamente significantes em nenhum dos domínios, quando comparada a qualidade de vida dos idosos dos três municípios entre si $(\mathrm{p}>0,05)$.

No entanto, ao se comparar A com os outros municípios, encontrou-se diferença estatisticamente significante no domínio ambiental, sugerindo melhor qualidade de vida dos idosos de $\mathrm{A}$, em relação aos de B e C $(p=0,039)$ (Tabela 2).

E, ao se comparar os idosos de A com os de C, encontrou-se diferença estatisticamente significante nos domínios social e ambiental, indicando melhor qualidade de vida dos idosos de $\mathrm{A}$, em relação aos de $\mathrm{C}(\mathrm{p}=0,044$ e 0,025 , respectivamente) (Tabela 2$)$.

As questões de autopercepção não mostraram diferença estatisticamente significante entre os idosos dos três municípios, em nenhuma das análises feitas ( $\mathrm{p}$ $>0,05)$ (Tabela 2).

Tabela 2. Distribuição de idosos matriculados em programas para a terceira idade de universidades localizadas no Estado de São Paulo, segundo domínios e questões de autopercepção sobre qualidade de vida, do instrumento WHOQOL - BREF

\begin{tabular}{|c|c|c|c|c|c|c|}
\hline Domínios & Municípios & Casos & $\begin{array}{c}\text { Rank } \\
\text { médio } 1^{1}\end{array}$ & $\begin{array}{c}\text { Rank } \\
\text { médio } 2^{2}\end{array}$ & $\begin{array}{c}\text { Rank } \\
\text { médio } 3^{3}\end{array}$ & $p$ \\
\hline \multirow{3}{*}{ Físico } & A & 106 & 95,56 & 95,56 & 78,84 & $0,436^{4}$ \\
\hline & $\mathrm{B}$ & 31 & 88,47 & 85,84 & & $0,217^{5}$ \\
\hline & $\mathrm{C}$ & 45 & 84,02 & 85,84 & 69,30 & $0,217^{6}$ \\
\hline \multirow{3}{*}{ Psicológico } & A & 106 & 95,78 & 95,78 & 79,99 & $0,192^{4}$ \\
\hline & $\mathrm{B}$ & 31 & 94,65 & 85,53 & & $0,192^{5}$ \\
\hline & $\mathrm{C}$ & 45 & 79,24 & 85,53 & 66,60 & $0,083^{6}$ \\
\hline \multirow{3}{*}{ Social } & A & 106 & 94,87 & 94,89 & 80,59 & $0,070^{4}$ \\
\hline & $\mathrm{B}$ & 31 & 101,45 & 86,77 & & $0,296^{5}$ \\
\hline & $\mathrm{C}$ & 45 & 76,66 & 86,77 & 65,19 & $0,044^{6}$ \\
\hline \multirow{3}{*}{ Ambiental } & A & 106 & 98,30 & 98,30 & 81,19 & $0,074^{4}$ \\
\hline & $\mathrm{B}$ & 31 & 89,11 & 82,02 & & $\mathbf{0 , 0 3 9 ^ { 5 }}$ \\
\hline & $\mathrm{C}$ & 45 & 77,13 & 82,02 & 63,77 & $\mathbf{0 , 0 2 5 ^ { 6 }}$ \\
\hline \multirow{3}{*}{$\begin{array}{l}\text { Autopercepção } \\
\text { sobre qualidade de } \\
\text { vida- Questão } 1\end{array}$} & A & 106 & 95,61 & 95,61 & 79,15 & $0,297^{4}$ \\
\hline & $\mathrm{B}$ & 31 & 89,87 & 85,77 & & $0,156^{5}$ \\
\hline & $\mathrm{C}$ & 45 & 82,94 & 85,77 & 68,59 & $0,120^{6}$ \\
\hline \multirow{3}{*}{$\begin{array}{l}\text { Autopercepção } \\
\text { sobre qualidade de } \\
\text { vida- Questão } 2\end{array}$} & A & 106 & 91,79 & 91,79 & 77,15 & $0,667^{4}$ \\
\hline & $\mathrm{B}$ & 31 & 97,13 & 91,10 & & $0,925^{5}$ \\
\hline & $\mathrm{C}$ & 45 & 89,94 & 91,10 & 73,30 & $0,595^{6}$ \\
\hline \multicolumn{7}{|c|}{${ }^{1}$ Comparação dos três municípios entre si } \\
\hline \multicolumn{7}{|c|}{${ }^{2}$ Comparação de A com B e C } \\
\hline \multicolumn{7}{|c|}{${ }^{3}$ Comparação de A com C } \\
\hline \multicolumn{7}{|c|}{${ }^{4}$ Kruskal Wallis Estatística qui-quadrado. Comparação dos três municípios entre si } \\
\hline \multicolumn{7}{|c|}{${ }^{5}$ Mann-Whitney Estatística Z. Comparação de A com B e C } \\
\hline${ }^{6}$ Mann-Whitney Est & ca Z. Co & A & & & & \\
\hline
\end{tabular}




\section{Discussão}

Neste estudo, foi investigada a influência do "desenvolvimento humano", medido pelo IDH e IDHM, sobre a qualidade de vida, medida pelo WHOQOL - BREF, de idosos frequentadores de Universidades Abertas para a Terceira Idade, em três municípios do Estado de São Paulo: São Caetano do Sul, São Paulo e Mogi das Cruzes, designados de A, $\mathrm{B}$ e $\mathrm{C}$, respectivamente.

A Organização Panamericana de Saúde, em convênio interagencial integrado pela Comissão Econômica para a América Latina e o Caribe (CEPAL), o Fundo de População das Nações Unidas (FNUAP), o Programa de Envelhecimento das Nações Unidas, a Organização Internacional do Trabalho (OIT) e o Banco Interamericano de Desenvolvimento (BID), e com a colaboração de diversos países da região, da América Latina e do Caribe, desenvolveu o Projeto SABE (Saúde, bemestar e envelhecimento) em sete países (Lebrão \& Duarte, 2003).

O SABE foi um dos primeiros esforços para coletar sistematicamente informações sobre as condições de vida de idosos, de ambos os gêneros, quanto a aspectos socioeconômicos, de saúde, redes de apoio, acesso aos serviços públicos etc. (Lebrão \& Duarte, 2003).

Nas sete cidades participantes do SABE, observou-se maior frequência de idosos com idades entre 65 e 74 anos, à semelhança do presente estudo. Constatou-se, também, que a proporção de idosos divorciados, separados e viúvos aumenta com a idade. Dada a disparidade na mortalidade entre homens e mulheres, em todas as idades as mulheres estão mais propensas a viverem sem cônjuge do que os homens (Albala et al., 2005).

Em Havana, por exemplo, quase 90\% das idosas e $43 \%$ dos idosos com 75 anos ou mais não possuem cônjuge (Albala et al., 2005), diferentemente do presente estudo, em que se observou maior frequência de idosos casados.

Em Montevidéu e Buenos Aires, mais de $25 \%$ das idosas vivem sozinhas, e em São Paulo e Havana, menos de $15 \%$. Nas outras cidades, esses valores são intermediários (Albala et al., 2005). Nos sete países participantes do $\mathrm{SABE}$, a proporção de idosos que vivem sozinhos é menor, em comparação aos países da Europa Ocidental e América do Norte, porém, maior, em comparação aos países da Ásia (Palloni, Devos, \& Pelaéz, 2000).
Quanto à escolaridade, em todos os países do $\mathrm{SABE}$ os idosos exibem nível mais elevado do que as idosas. Em Havana, independentemente do gênero e da idade, mais de $50 \%$ dos idosos completaram o ensino superior, e em São Paulo, tal número é inferior a 20\% (Albala et al., 2005). No presente estudo, notouse maior frequência de idosos que completaram o ensino médio, em comparação ao ensino fundamental e ao ensino superior.

O trabalho de Lebrão e Laurenti (2005), "Saúde, bem-estar e envelhecimento: o estudo SABE no município de São Paulo", à semelhança do presente estudo, mostrou maioria de idosos do gênero feminino (58,6\%), com idade média de 68 anos. Também, a maioria dos idosos vivia acompanhada $(86,8 \%)$, possuía renda familiar mensal média de 2,1 salários mínimos, não trabalhava $(71,4 \%)$ e estava aposentada $(71,1 \%)$.

No trabalho de Lebrão e Duarte (2003), "O projeto SABE no município de São Paulo: uma abordagem inicial", observou-se que a maioria dos idosos estava casada $(56,9 \%)$, como no presente estudo.

A pesquisa "Idosos no Brasil - Vivências, desafios e expectativas na terceira idade", produzida por iniciativa conjunta do SESC (Serviço Social do Comércio) Nacional e SESC São Paulo e da FPA (Fundação Perseu Abramo), em abril de 2006, levantou dados sócio-demográficos, sobre saúde, inserção social, aspectos selecionados da qualidade de vida percebida e atitudes em relação à velhice e juventude (Neri, 2007b).

Envolveu amostra probabilística de 2136 idosos, com 60 anos e mais, e 1608 jovens e adultos, de 16 a 59 anos, residentes em 204 municípios das cinco macrorregiões do país (Sudeste, Nordeste, Norte, Sul e Centro-Oeste) (Neri, 2007b).

$\mathrm{Na}$ referida pesquisa, como no presente estudo, verificou-se predomínio de idosos do gênero feminino em relação ao gênero masculino $(57 \%$ e $43 \%$, respectivamente). Ainda, constatou-se que $86 \%$ das idosas e $66 \%$ dos idosos não trabalhavam, e que havia mais homens aposentados que não trabalhavam $(61 \%)$ do que mulheres $(46 \%)$. Isso reflete a tendência cultural própria da época, em que bem menos mulheres do que hoje trabalhavam fora de casa, e pouquíssimas desenvolveram carreira que as conduzisse à aposentadoria (Neri, 2007b).

A pesquisa revelou que $32 \%$ dos idosos e $28 \%$ das idosas possuíam renda familiar mensal de mais de dois a cinco salários mínimos (Neri, 2007b), intervalo de renda próximo ao do presente estudo. 
Quanto à escolaridade, a pesquisa mostrou que $18 \%$ dos idosos nunca frequentaram uma escola, $71 \%$ tinham o ensino fundamental, $7 \%$ tinham o ensino médio e apenas $4 \%$ dos idosos mencionaram o curso superior (Neri, 2007b). Tais dados diferem do presente estudo, em que houve maior frequência de idosos que completaram o ensino médio.

Em relação ao estado marital, 52\% dos idosos eram casados, $6 \%$ eram solteiros, $34 \%$ eram viúvos e $8 \%$, divorciados. Quanto à posse de filhos, $94 \%$ dos idosos disseram ter pelo menos um filho (Neri, 2007b). Esses dados são semelhantes aos do presente estudo, em que se observou maior frequência de idosos casados e com pelo menos um filho.

No que tange à quantidade de pessoas com quem vivem de forma permanente, $85 \%$ dos idosos viviam com pelo menos uma pessoa (Neri, 2007b), resultado semelhante ao do presente estudo, em que a maioria dos idosos vivia acompanhada.

Com respeito aos domínios e às questões de autopercepção sobre qualidade de vida dos idosos nos três municípios estudados, constatou-se significância estatística no domínio ambiental, quando se comparou A com B e C, e nos domínios social e ambiental, ao se comparar apenas A com C. Nos domínios físico e psicológico e nas duas questões de auto- percepção, as diferenças encontradas não foram estatisticamente significantes em nenhuma das análises feitas.

O domínio social do WHOQOL - BREF engloba o apoio social e a atividade sexual. $O$ domínio ambiental, por sua vez, concerne a cuidados de saúde, segurança física, ambiente no lar, recursos financeiros, informação, recreação e lazer, ambiente físico e transporte (Fleck et al., 2008).

O domínio físico engloba dor e desconforto, energia e fadiga, sono e repouso, mobilidade, atividades da vida cotidiana, dependência de medicação e capacidade de trabalho. O domínio psicológico refere-se a sentimentos positivos, pensar, aprender, autoestima, imagem corporal, sentimentos negativos e espiritualidade (Fleck et al., 2008).

O IDHM destaca três índices para avaliação e posicionamento dos municípios no ranking nacional que são, justamente, os descritos e analisados neste estudo: renda, educação e longevidade. Ao analisar os dados da caracterização dos idosos, vale ressaltar alguns indicadores sociais que permitem compreender a relação entre o IDHM e os resultados no inventário de qualidade de vida: apenas 5,5\% tinham idade $\geq 80$ anos, na faixa etária da longevidade; a maioria era casada, tinha filhos e não morava sozinha; o nível educacional da maioria era do ensino médio ao superior; a renda familiar mensal de praticamente metade dos idosos era $\geq$ três salários mínimos; a maioria estava aposentada e não trabalhava.

Portanto, os idosos do presente estudo revelaram recursos sociais, educacionais e financeiros que podem justificar a elevada pontuação obtida nos diferentes domínios avaliados no WHOQOL - BREF, especialmente pelos habitantes do município A, cujo IDHM é o maior do país.

É notório que os determinantes sociais impactaram na trajetória de vida dos idosos dos três municípios estudados, sendo que o tipo de ambiente e a sociedade na qual o idoso está inserido colaboram na manutenção e promoção do bem-estar do idoso no seu processo de envelhecimento, o qual é um objeto e uma área de estudo de extrema complexidade em virtude das diversas contingências de vida (Bassit \& Witter, 2010; Guariento \& Neri, 2011; Neri, 2011; Witter, Buriti, \&Witter, 2011).

Entretanto, as disparidades na qualidade de vida dos três municípios não foram percebidas pelos idosos, o que pode ser observado nos resultados obtidos nas duas questões de autopercepção, que não mostraram diferenças estatisticamente significantes entre os grupos.

De acordo com Pereira (2002), as avaliações subjetivas da qualidade de vida em idosos devem considerar o que acontece ao indivíduo nas diferentes etapas do envelhecimento, desde mudanças físicas até a desvalorização social, consequente da aposentadoria, considerando qual seu sentimento e entendimento dessas situações, seus ganhos e perdas psicológicas, suas frustrações e aspirações.

Nesse sentido, segundo Santos, Santos, Fernandes e Henriques (2002), a qualidade de vida do idoso compreende diversos critérios de natureza biológica, psicológica e socioestrutural, uma vez que vários elementos são apontados como determinantes ou indicadores de bem-estar na velhice: longevidade, saúde biológica, saúde mental, satisfação, controle cognitivo, competência social, produtividade, eficácia cognitiva, status social, continuidade de papéis familiares e ocupacionais e continuidade de relações informais com amigos.

A família é uma rede social importante na manutenção e promoção das atividades e do convívio sociais, na medida em que proporciona a interação interpessoal entre os familiares, outras pessoas e o idoso, o que permite o estabelecimento de relações intergeracionais que podem colaborar com o envelhecimento saudável (Witter \& Camilo, 2011). 
Pereira et al. (2006) estudaram a influência de gênero, idade, estado civil, nível de escolaridade e renda nos domínios do WHOQOL - BREF. Essas variáveis sociodemográficas não exerceram influência na qualidade de vida global dos idosos, com exceção da variável gênero, que apresentou escores médios significantemente maiores entre os homens nos domínios físico, psicológico e ambiental.

Sprangers et al. (2000), todavia, enfatizaram que fatores como idade avançada, gênero feminino, baixo nível de escolaridade e não ter companheiro estão relacionados a baixos níveis de qualidade de vida.

Conforme Jakobsson, Hallberg e Westergren (2004), uma boa situação socioeconômica mostra-se associada à melhor qualidade de vida. Dessa forma, o fato de os municípios A e B apresentarem rendas per capita superiores às do município $\mathrm{C}$ pode contribuir para que seus habitantes tenham melhor qualidade de vida do que os residentes desse último município.

No estudo de Pereira et al. (2006), ao se analisar a contribuição dos diferentes domínios na qualidade de vida global dos idosos, verificou-se que a contribuição dos quatro domínios juntos foi de $36,1 \%$, e que os domínios diferiram a respeito da contribuição individual na qualidade de vida global. O domínio que mais contribuiu na qualidade de vida global foi o físico, seguido do ambiental e do psicológico, respectivamente, e o domínio social não teve contribuição estatisticamente significante, diferentemente do presente estudo.

Segundo a OMS (2001), o ambiente físico em que o idoso está inserido pode determinar a dependência ou não do indivíduo. Nesse sentido, idosos que vivem em ambientes inseguros são menos propensos a saírem sozinhos e, portanto, estão mais susceptíveis ao isolamento e à depressão, bem como a ter mais problemas de mobilidade e pior estado físico, o que compromete sua qualidade de vida.

O'Shea (2003) corrobora o que diz a OMS (2001), ao afirmar que a moradia e o ambiente físico adequados têm influência positiva na qualidade de vida do idoso.

Em relação à segurança, que se refere ao domínio ambiental, é interessante observar que B, comparado aos outros dois municípios estudados, é o que apresenta maiores índices de violência, o que, teoricamente, refletiria negativamente na qualidade de vida de seus habitantes. Contudo, B apresenta IDHM superior ao de $\mathrm{C}$, embora inferior ao de $\mathrm{A}$.

Assim, provavelmente, há uma compensação mais positiva para $B$ nos outros itens que compõem o domínio ambiental, tais como: condições de saúde (acesso a tratamentos, hospitais etc), possibilidade de lazer e recreação, renda familiar e possibilidade de atividades profissionais para idosos.

\section{Conclusão}

A partir dos resultados obtidos, concluiu-se que o "desenvolvimento humano", medido pelo IDH e IDHM, exerceu influência sobre a qualidade de vida dos idosos, medida pelo WHOQOL - BREF, nos domínios social e ambiental, sendo que os melhores resultados foram obtidos entre os habitantes de A (município que exibe o maior IDHM do país) e B, em comparação aos de $\mathrm{C}$ (município que exibe o menor IDHM dentre os estudados), confirmando-se a hipótese inicial dos autores.

É importante destacar que os idosos estudados frequentavam programas para a terceira idade oferecidos por Universidades Abertas para a Terceira Idade em seus respectivos municípios, o que os diferencia dos idosos da população em geral, no sentido de acesso e busca por conhecimento e cuidados com a saúde. Assim, seria esperado que eles tivessem uma melhor percepção de sua qualidade de vida e do ambiente que os cerca.

Entretanto, os idosos não perceberam o quanto os fatores relacionados ao ambiente onde vivem, incluindo segurança física, recursos financeiros, acesso aos serviços de saúde, oportunidades em adquirir novas informações, oportunidades de atividades de lazer, transporte e ambiente físico (poluição/ruído/trânsito/ clima), e itens referentes às suas relações pessoais, incluindo o apoio social que recebem, influenciaram sua qualidade de vida, e isso pode ser visto nos resultados obtidos nas duas questões de autopercepção, que não mostraram diferenças estatisticamente significantes entre os grupos, sugerindo a possibilidade de uma barreira para a busca de políticas que promovam melhor qualidade de vida em seus respectivos municípios.

\section{Nota}

WHO Quality of Life

\section{Referências}

Albala, C., Lebrão, M. L., León Díaz, E. M., Ham-Chande, R., Hennis, A. J., Palloni, A. et al. (2005). Encuesta Salud, Bienestar y Envejecimiento (SABE): metodología de la encuesta y perfil de la poblacíon estudiada. Revista Panamericana de Salud Publica, 17(5-6), 307-322. 
Bassit, A. Z. \& Witter, C. (2010). Envelhecimento: objeto de estudo e campo de intervenção. In G. P. Witter (Org.), Envelhecimento: referenciais teóricos e pesquisas (2a ed., pp. 15-31). Campinas, SP: Alínea.

Barros, R. P., Henriques, R., \& Mendonça, R. (2001). A estabilidade inaceitável: desigualdade e pobreza no Brasil (Texto para Discussão, n. 800). Rio de Janeiro: IPEA.

Fleck, M. P. A. et al. (2008). A avaliação de qualidade de vida: guia para profissionais da saúde. Porto Alegre: Artmed.

Grupo de Estudos em Qualidade de Vida. Departamento de Psiquiatria e Medicina Legal. UFRGS. (2008). Versão em português dos Instrumentos de Avaliação de Qualidade de Vida (WHOQOL) 1998. Porto Alegre: Autor. Acesso em 05 de agosto, 2014, em http://www.ufrgs.br/psiquiatria/psiq/ whoqol.html

Guariento, M. H. \& Neri, A. L. (Orgs.). (2011). Fragilidade, saúde e bem-estar em idosos. Dados do Estudo Fibra. Campinas, SP: Alínea.

Instituto Brasileiro de Geografia e Estatística - IBGE. (2010). Acesso em: 29 de março, 2014, em http://www.cidades.ibge. gov.br/xtras/temas.php?lang $=\&$ codmun $=355030 \&$ idtema $=1$ $6 \&$ search $=$ sao-paulo|sao-paulo/sintese-das-informacoes

Jakobsson, U., Hallberg, I. R., \& Westergren, A. (2004). Overall and health related quality of life among the oldest old in pain. Quality of Life Research, 13(1), 125-36.

Lebrão, M. L. \& Duarte, Y. A. O. (2003). O projeto SABE no município de São Paulo: uma abordagem inicial. Acesso em 02 de maio, 2011, em http://bvsms.saude.gov.br/bvs/ publicacoes/1_saber.pdf

Lebrão, M. L. \& Laurenti, R. (2005). Saúde, bem-estar e envelhecimento: o estudo SABE no município de São Paulo. Revista Brasileira de Epidemiologia, 8(2), 127-141. Acesso em 02 de maio, 2011, em http://www.fsp.usp.br/ $\underline{\text { sabe/Extras/Artigos/O_Estudo_SABE_no_municipio_de }}$ Sao Paulo.pdf

Neri, A. L. (Org.). (2007a). Qualidade de vida na velhice: enfoque multidisciplinar. Campinas, SP: Alínea.

Neri, A. L. (Org.). (2007b). Idosos no Brasil - Vivências, desafios e expectativas na terceira idade. São Paulo: Fundação Perseu Abramo / SESC-SP.

Neri, A. L. (2011). Teorias Psicológicas do envelhecimento: percurso histórico e teorias atuais. In E. V. Freitas \& F. A. Xavier (Orgs.), Tratado de Geriatria e Gerontologia (pp. 3546). Rio de Janeiro: Guanabara.

Organización Mundial de la Salud - OMS. (2001). Salud y envejecimiento: un documento para el debate. Versión preliminar. Boletín sobre el Envejecimiento: Perfiles $y$ Tendencias, 4-5. Acesso em 28 de agosto, 2011, em http://www.sld.cu/galerias/pdf/sitios/gericuba/perfiles y tendencias.pdf

O'Shea, E. (2003). La mejora de la calidad de vida de las personas mayores dependientes. Boletín sobre el Envejecimiento: Perfiles y Tendencias, 6. Acesso em 28 de agosto, 2011, em http://envejecimiento.csic.es/documentos/ documentos/boletinsobreenvejec06.pdf

Palloni, A., Devos, S., \& Peláez, M. (2000). Aging in Latin America and the Caribbean. Washington, D. C.: PAHO Working Papers.

Pereira, D. E. C. (2002). Qualidade de vida na terceira idade e sua relação com o trabalho no grupo de terceira idade "Amor e Carinho" de Santa Terezinha de Itaipu - PR. Dissertação de Mestrado, Programa de Pós-Graduação em
Engenharia de Produção, Universidade Federal de Santa Catarina, Florianópolis.

Pereira, R. J., Cotta, R. M. M., Franceschini, S. C. C., Ribeiro, R. C. L., Sampaio, R. F., Priore, S. E. et al. (2006). Contribuição dos domínios físico, social, psicológico e ambiental para a qualidade de vida global de idosos. Revista de Psiquiatria do Rio Grande do Sul, 28(1), 27-38. Acesso em 28 de agosto, 2011, em http://www.scielo.br/scielo.php?pid=S0101$\underline{81082006000100005 \& \text { script }=\text { sci_arttext }}$

Pinheiro, J. C. V., Carvalho, R. M., \& Freitas, K. S. (2009). Análise do suprimento atual e potencial de água potável para os municípios cearenses. Sociedade \& Natureza, 21(2), 107121.

Programa das Nações Unidas para o Desenvolvimento - PNUD. (2003). Atlas do Desenvolvimento Humano do Brasil. Acesso em 10 de abril, 2011, em http://www.pnud.org.br/IDH/ Default.aspx?indiceAccordion=1\&li=li AtlasMunicipios

Programa das Nações Unidas para o Desenvolvimento PNUD. (2006). Brasil melhora IDH, mas cai no ranking. Acesso em 10 de abril, 2011, em http://www.pnud.org.br/ HDR/arquivos/rdh2006/rdh2006 IDH.pdf

Programa das Nações Unidas para o Desenvolvimento - PNUD. (2010). Brasil ocupa $73^{a}$ posição entre 169 países no IDH 2010. Acesso em 22 de junho, 2011, em http://g1.globo.com/ brasil/noticia/2010/11/brasil-ocupa-73-posicao-entre-169paises-no-idh-2010.html

Programa das Nações Unidas para o Desenvolvimento - PNUD. (2012). Desenvolvimento Humano e IDH. Acesso em 05 de agosto, 2014, em http://www.pnud.org.br/IDH/DH.aspx

Programa das Nações Unidas para o Desenvolvimento - PNUD, Instituto de Pesquisa Econômica Aplicada IPEA, \& Fundação João Pinheiro - FJP. (2012). Atlas do Desenvolvimento Humano no Brasil 2013. Acesso em 29 de março, 2014, em http://www.atlasbrasil.org.br/2013/o_atlas/ idhm

Santos, S. R., Santos, I. B. C., Fernandes, M. G. M., \& Henriques, M. E. R. M. (2002). Qualidade de vida do idoso na comunidade: aplicação da escala de Flanagan. Revista Latino-Americana de Enfermagem, 10(6), 757-764.

Scarpin, J. E. \& Slomski, V. (2007). Estudo dos fatores condicionantes do índice de desenvolvimento humano nos municípios do estado do Paraná: instrumento de controladoria para a tomada de decisões na gestão governamental. Revista de Administração Pública, 41(5), 909-933.

Sen, A. K. (2000). Desenvolvimento como liberdade. São Paulo: Companhia das Letras.

Siegel, S. \& Castellan Jr., N. J. (2006). Estatística nãoparamétrica para ciências do comportamento ( $2^{\mathrm{a}} \mathrm{ed}$.). Porto Alegre: Artmed.

Silva, O. M. P. \& Panhoca, L. (2007). A contribuição da vulnerabilidade na determinação do índice de desenvolvimento humano: estudando o estado de Santa Catarina. Ciência \& Saúde Coletiva, 12(5), 1209-1219.

Sprangers, M. A., De Regt, E. B., Andries, F., Van Agt, H. M., Bijl, R. V., De Boer, J. B. et al. (2000). Which chronic conditions are associated with better or poorer quality of life? Journal of Clinical Epidemiology, 53(9), 895-907.

Torres, H. G., Ferreira, M. P., \& Dini, N. P. (2003). Indicadores sociais: por que construir novos indicadores como o IPRS. São Paulo em Perspectiva, 17(3-4), 80-90.

Vecchia, R. D., Ruiz, T., Bocchi, S. C. M., \& Corrente, J. E. (2005). Qualidade de vida na terceira idade: um conceito 
subjetivo. Revista Brasileira de Epidemiologia, 8(3), 246252.

Witter, C. \& Camilo, A. R. (2011). Família e envelhecimento. In C. Witter, M.A. Buriti, \& G. P. Witter(Orgs.), Envelhecimento e contingências de vida (pp. 83-102). Campinas, SP: Alínea.

Witter, C., Buriti, M. A., \& Witter, G. P. (Orgs.). (2011). Envelhecimento e contingências de vida. Campinas, SP: Alínea.

Submissão em: 14/10/2011

Revisão em: 30/01/2014

Aceite em: 04/03/2014

Nathaly Wehbe Dawalibi é Nutricionista e Mestre em Ciências do Envelhecimento pela Universidade São Judas Tadeu. Endereço: Rua dos Ingleses, 454, ap 71. Morro dos Ingleses. São Paulo/SP, Brasil. CEP 01329-000.

(A/C: Nathaly Wehbe Dawalibi) E-mail: nathalydawalibi@yahoo.com.br

Rita Maria Monteiro Goulart é Nutricionista, doutora em Saúde Pública, docente no curso de mestrado em Ciências do Envelhecimento. E-mail: ritagoulart@yahoo.com.br

Rita de Cássia de Aquino é Nutricionista, doutora em Nutrição e Saúde Pública, docente no curso de mestrado em Ciências do Envelhecimento da Universidade São Judas Tadeu, São Paulo/SP, Brasil. E-mail: rcaquino@uol.com.br
Carla Witter é Doutora em Psicologia Escolar e do Desenvolvimento Humano, docente no curso de mestrado em Ciências do Envelhecimento da Universidade São Judas Tadeu. São Paulo/SP, Brasil. E-mail: cwitter@uol.com.br

Marcelo de Almeida Buriti é Doutor em Psicologia (Profissão e Ciência), docente no curso de mestrado em Ciências do Envelhecimento da Universidade São Judas Tadeu. São Paulo/SP, Brasil. E-mail: marceloburiti@hotmail.com

Leandro Campi Prearo é Professor de Estatística, doutor em Administração (Métodos Quantitativos). Universidade Municipal de São Caetano do Sul - USCS. São Caetano/SP, Brasil. E-mail: leandro.prearo@uscs.edu.br

\section{Como citar:}

Dawalibi, N. W., Goulart, R. M. M., Aquino, R. C., Witter, C., Buriti, M. A., \& Prearo, L. C. (2014). Índice de desenvolvimento humano e qualidade de vida de idosos frequentadores de universidades abertas para a terceira idade. Psicologia \& Sociedade, 26(2), 496-505. 